\title{
Simaomicin $\alpha$ : Effects on the Cell Cycle of Synchronized, Cultured Plasmodium falciparum
}

\author{
Aki Ishiyama, Kazuhiko Otoguro, Miyuki Namatame, Aki Nishihara, Toshiaki Furusawa, \\ Yoko Takahashi, Rokuro Masuma, Kazuro Shiomi, Satoshi Ōmura
}

Received: February 20, 2008 / Accepted: March 31, 2008

(C) Japan Antibiotics Research Association

\begin{abstract}
Simaomicin $\alpha$ shows potent antimalarial activity in vitro and is known to be a cell-cycle effector. As erythrocytic schizogony of Plasmodium correlates with cell cycle events, we investigated the effect of simaomicin $\alpha$ on stage development of the malaria parasite Plasmodium falciparum. Simaomicin $\alpha$ interferes with normal parasite development in a time and concentration dependent manner. Parasites exposed to $2.5 \mathrm{nM}$ simaomicin $\alpha$ at the ring stage or trophozoite stage showed disrupted development and immature schizont-like and segmenterlike forms were observed. However, schizont stage parasites were not affected by $2.5 \mathrm{nM}$ simaomicin $\alpha$. It is unclear whether mitosis involved in sequential parasite development occurred when parasites were exposed to simaomicin $\alpha$ at the ring or trophozoite stage. At a concentration of $5.0 \mathrm{nM}$, simaomicin $\alpha$ inhibited merozoite-trophozoite development. This concentration curtails p-LDH activity at all parasite stages, although its impact on the schizont stage is delayed for 24 hours.
\end{abstract}

Keywords Simaomicin $\alpha$, Plasmodium, malaria, cellcycle, synchronous culture

During the course of our long-term and continuing programme of screening microbial metabolites, we have discovered various compounds that exhibit potent antimalarial activities $[1 \sim 6]$. We previously reported that simaomicin $\alpha$ showed potent antimalarial activity in vitro [6]. Furthermore, we reported that it has bleomycininduced G2 checkpoint inhibitory activity against human Tcell leukemia-derived Jurkat cells [7].

Erythrocytic schizogony of Plasmodium falciparum correlates with specific cell-cycle events, such as G1, S and $\mathrm{M}$ phases. However, several aspects remain poorly understood, particularly in connection with the G2 and M phases $[8,9]$. In the present study we demonstrate the stage-specific antimalarial activity of simaomicin $\alpha$, although the mechanism of action remains unresolved. Consequently, simaomicin $\alpha$ may be useful in studying the cell-cycle of $P$. falciparum.

The drug-sensitive $P$. falciparum strain FCR3 was cultured and synchronized using the D-sorbitol method [10] to produce specific parasite stages. At 24 36 hours after the first synchronization, the parasites were synchronized again. At 36 hours after the second synchronization the last synchronization was completed. The cells were treated and then used for experimentation. Two or three D-sorbitol treatments were carried out to prepare ring-stage parasites $(>90 \%)$ based on direct observation of parasite growth. For experimental purposes, parasite stages were determined as follows: Ring stage ( $0 \sim 12$ hours after last synchronization) which has a ring form; early-trophozoite stage (12 24
A. Ishiyama, K. Otoguro (Corresponding author), M. Namatame, A. Nishihara, T. Furusawa: Research Center for Tropical Disease, The Kitasato Institute, 5-9-1 Shirokane, Minatoku, Tokyo 108-8641, Japan,

E-mail: otoguro@lisci.kitasato-u.ac.jp
S. Ōmura, Y. Takahashi, R. Masuma, K. Shiomi: Kitasato Institute for Life Sciences and Graduate School of Infection Control Sciences, Kitasato University, 5-9-1 Shirokane, Minatoku, Tokyo 108-8641, Japan 
hours after the last synchronization), which has a large or thick ring appearance with no pigment; trophozoite stage (24 36 hours after final synchronization), which has an even cytosolic component and pigment with a single nucleus; schizont stage (36 44 hours after final synchronization), which has two or more nuclei; and the merozoite stage (44 47 hours after final synchronization) which exhibits a post-mitotic form.

In the present study, ring-stage parasites with $2.0 \%$ hematocrit and $2 \sim 3 \%$ parasitaemia were cultured in 12 well plates, and $50 \mu \mathrm{l}$ of simaomicin $\alpha$ or chloroquine, at various concentrations, were added at $0,12,24,36$ and 46 hours after the final D-sorbitol treatment that corresponded to each parasite development stage. Parasite lactate dehydrogenase ( $\mathrm{p}-\mathrm{LDH})$ activity was evaluated, and morphological observation of the parasite thin smear was carried out every 24 hours.

It is believed that p-LDH activity corresponds with parasite development and parasitaemia. Control parasites exhibited changing p-LDH activity in a stepwise fashion (Fig. 1), as p-LDH levels are different at each development stage; ring stage $<$ trophozoite stage $<$ schizont stage. Direct observation confirmed that the changes were caused by lack of development rather than parasite death.
In general, $5.0 \mathrm{nM}$ simaomicin $\alpha$ had an immediate impact on parasite development, as measured by $\mathrm{p}-\mathrm{LDH}$ activity (Fig. 1A D). At the ring and early-trophozoite stages, this concentration interfered with parasite development for the duration of the experiment. In both the trophozoite and schizont stage $5.0 \mathrm{nM}$ simaomicin $\alpha$ curtailed the rate of increase of $\mathrm{p}-\mathrm{LDH}$ activity for the first 24 hours after which it caused a marked and lasting reduction. In comparison, $2.5 \mathrm{nM}$ simaomicin $\alpha$ showed no impact on either ring or early-trophozoite stages for at least 24 hours. Thereafter, p-LDH began to be decreased compared with controls and, after $24 \sim 48$ hours, significantly reduced. This pattern was also evident in the trophozoite stage, where a decrease in p-LDH activity was also seen after 24 hours. However, simaomicin at this concentration had no effect on the schizont stage.

Using chloroquine $(100 \mathrm{nM}), \mathrm{p}-\mathrm{LDH}$ did not increase significantly for the ring, early-trophozoite and trophozoite stages (Fig. 1E, F and G), but exposure at the schizont stage increased p-LDH activity and resulted in normal but delayed development (Fig. 1H).

Morphological changes of the control parasites, illustrating normal development in the life cycle, are shown in Fig. 2A. When ring-stage parasites were exposed to

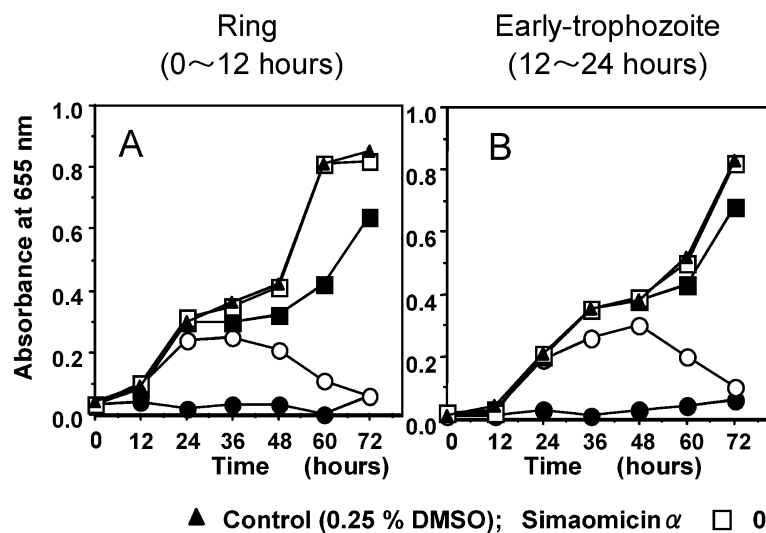

Trophozoite
$(24 \sim 36$ hours $)$

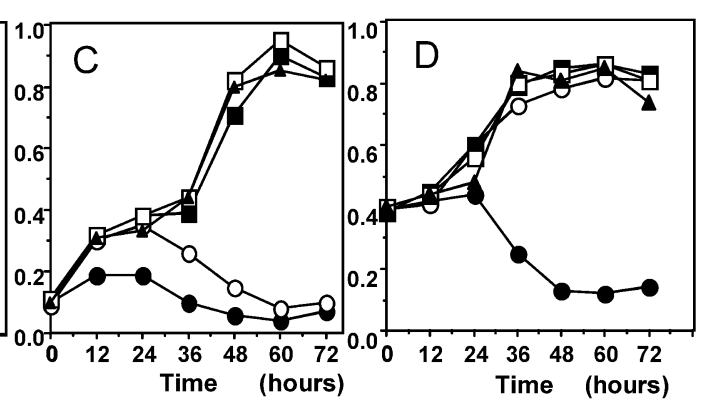

Schizont

(36 42 hours)

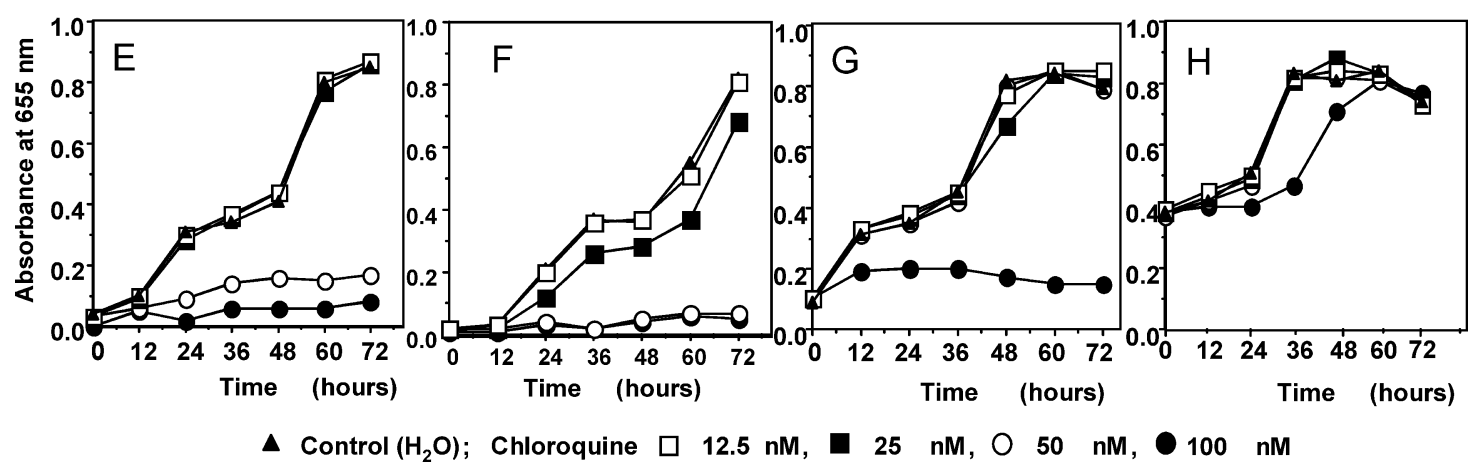

Fig. 1 Time course of p-LDH activity of different parasite stages cultured with various concentrations of simaomicin $\alpha$ or chloroquine: ring ( $A$ and $E$ ), early-trophozoite ( $B$ and $F)$, trophozoite $(C$ and $G$ ) and schizont $(D$ and $H)$ stages. 


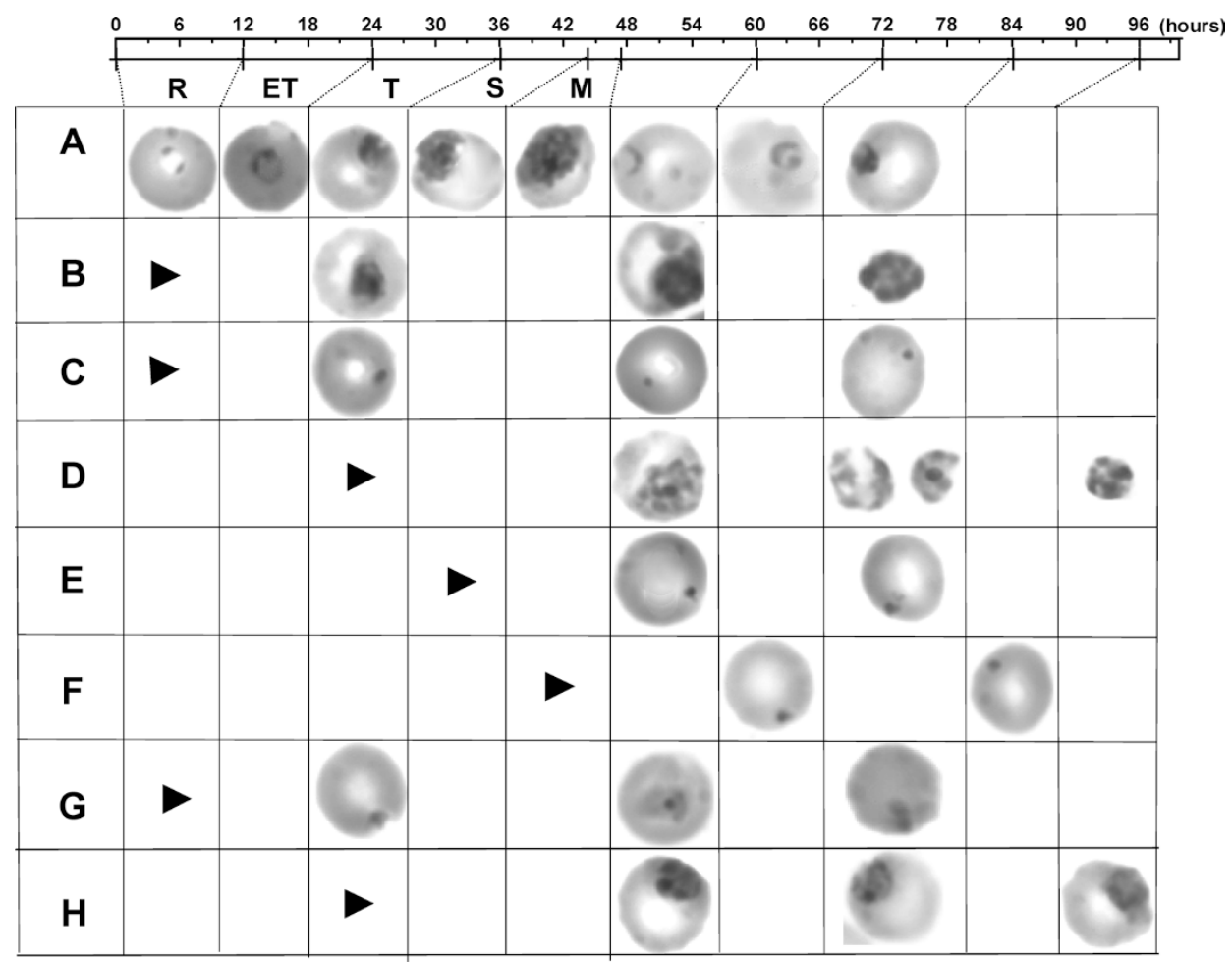

Fig. 2 Morphological observation of parasites exposed to 2.5 or $5.0 \mathrm{nM}$ simaomicin $\alpha$ and $100 \mathrm{nM}$ chloroquine.

DMSO $(0.25 \%)$ or $\mathrm{H}_{2} \mathrm{O}$ were used as controls. A. control parasites; B. simaomicin $\alpha(2.5 \mathrm{nM})$ treated ring stage; C. simaomicin $\alpha$ $(5 \mathrm{nM})$ treated ring stage; D. simaomicin $\alpha(2.5 \mathrm{nM})$ treated trophozoite stage; E. simaomicin $\alpha(5.0 \mathrm{nM})$ treated schizont stage; F. simaomicin $\alpha(5.0 \mathrm{nM})$ treated merozoite stage; G. chloroquine $(100 \mathrm{nM})$ treated ring stage; H. chloroquine $(100 \mathrm{nM})$ treated trophozoite stage. Arrows indicate point of drug treatment. $\mathrm{R}=$ ring stage; $\mathrm{ET}=$ early-trophozoite stage; $\mathrm{T}=$ trophozoite stage; $\mathrm{S}=$ schizont stage; $\mathrm{M}=$ merozoite stage.

$2.5 \mathrm{nM}$ simaomicin $\alpha$, they developed into the trophozoite stage but after 48 hours incubation, only immature schizont-like forms followed by the segmenter-like forms were observed (Fig. 2B). The same phenomenon was observed when trophozoite-stage parasites were treated with $2.5 \mathrm{nM}$ simaomicin $\alpha$ (Fig. 2D) and when earlytrophozoite stage parasites were treated with $2.5 \mathrm{nM}$ simaomicin $\alpha$ (data not shown). In comparison, when schizont stage parasites were exposed to $2.5 \mathrm{nM}$ simaomicin $\alpha$, normal life-cycle stage development and red blood cell re-invasion were observed (data not shown). Shrunken parasites, appearing as a dot in the erythrocyte, were seen when ring-stage, schizont-stage and merozoitestage parasites were exposed to $5.0 \mathrm{nM}$ of simaomicin $\alpha$ (Fig. 2C, E and F), with similar results being seen in earlytrophozoite stage parasites treated with $5.0 \mathrm{nM}$ simaomicin $\alpha$ (data not shown). In the case of treatment with $100 \mathrm{nM}$ chloroquine, ring-stage and trophozoite-stage parasites did not develop (Fig. 2G, H).

It is understood that the cell-cycle of Plasmodium spp. is as follows. G0-like phase is a merozoite (both free and late segmenters) form; G1 phase is an early trophozoite stage; $\mathrm{S}$ phase is soon after the appearance of pigmented trophozoites (this phase is considered as the trophozoite stage in this experiment); $\mathrm{M}$ phase is after the first nuclear division (this is considered as the schizont stage in this experiment); and the $\mathrm{G} 2$ phase, which is still unclear $[8,9]$. Naughton and Bell reported that drugs such as Hoechst 33342, roscovitine and L-mimosine blocked the trophozoite-schizont boundary, presumably in the S or G2 phase. These drugs, especially roscovitine, blocked nucleus division, preventing development from trophozoite to schizont [11].

Simaomicin $\alpha$ affects $P$. falciparum development in a time and concentration dependent manner. Our results indicate that $2.5 \mathrm{nM}$ simaomicin $\alpha$ acts at the trophozoiteschizont interface (corresponding to the S-M phase), and does not act at the schizont-new ring boundary (corresponding to the M-G1 phase). Simaomicin $\alpha$ at $5 \mathrm{nM}$ concentration acts after re-inversion to the new erythrocytering stage (corresponding to the G0-G1 phase), as well as affecting development at the ring-trophozoite transition, 
corresponding to the G0, G1-S phase.

The impact of simaomicin $\alpha$ is morphologically very different from that of chloroquine. Both ring- and trophozoite-stage parasites exposed to simaomicin $\alpha$ seem to undergo mitosis, in contrast to the normal G2 phase inhibition. However, it is unclear whether normal mitosis that occurs during sequential parasite development is disrupted by simaomicin a exposure.

The target of simaomicin $\alpha$ and the mode of action remain unsubstantiated. The compound does not have a blocking effect on known G2 inhibitors, such as Chk1, Chk2, ATM, ATR and Weel, in Jurkat cells [7]. We further observed that bleomycin at $8.8 \mu \mathrm{M}$ has no effect on parasite growth and also did not exhibit any synergic or additive effects (data not shown).

Research on the simaomicin $\alpha$ target needs to be carried out, and it would be interesting to determine if simaomicin $\alpha$ has specific and unique activity on the Plasmodium cell cycle, as it might prove to be a useful tool for detailed analysis of the parasite's cellular growth and development.

Acknowledgements This work was supported in part by funds from the UNICEF/UNDP/World Bank/WHO Special Programme for Research and Training in Tropical Disease (grants ID 990806 and ID A10124), and a grant for All Kitasato Project Study (AKPS). We also thank Miss H. Sekiguchi of the Kitasato Institute for the antimalarial assay.

\section{References}

1. Otoguro K, Kohana A, Manabe C, Ishiyama A, Ui H, Shiomi K, Yamada H, Ōmura S. Potent antimalarial activities of the polyether antibiotic, X-206. J Antibiot 54: 658-663 (2001)

2. Otoguro K, Ishiyama A, Ui H, Kobayashi M, Manabe C,
Yan G, Takahashi Y, Tanaka H, Yamada H, Ōmura S. In vitro and in vivo antimalarial activities of the monoglycoside polyether antibiotic, K-41, against drug-resistant strains of Plasmodia. J Antibiot 55: 832-834 (2002)

3. Otoguro K, Ui H, Ishiyama A, Arai N, Kobayashi M, Takahashi Y, Masuma R, Shiomi K, Yamada H, Ōmura S. In vitro antimalarial activities of the microbial metabolites. J Antibiot 56: 322-324 (2003)

4. Otoguro K, Ui H, Ishiyama A, Kobayashi M, Togashi H, Takahashi Y, Masuma R, Tanaka H, Tomoda H, Yamada H, Ōmura $\mathrm{S}$. In vitro and in vivo antimalarial activities of a non-glycosidic 18-membered macrolide antibiotic, borrelidin, against drug-resistant strains of Plasmodia. J Antibiot 56: 727-729 (2003)

5. Otoguro K, Ishiyama A, Kobayashi M, Sekiguchi H, Izuhara T, Sunazuka T, Tomoda H, Yamada H, Ōmura S. In vitro and in vivo antimalarial activities of a carbohydrate antibiotic, prumycin, against drug-resistant strains of Plasmodia. J Antibiot 57: 400-402 (2004)

6. Ui H, Ishiyama A, Sekiguchi H, Namatame M, Nishihara A, Takahashi Y, Shiomi K, Otoguro K, Ōmura S. Selective and potent in vitro antimalarial activities found in four microbial metabolites. J Antibiot 60: 220-222 (2007)

7. Arai M, Sato H, Kobayashi H, Suganuma M, Kawabe T, Tomoda H, Ōmura S. Selective inhibition of bleomycininduced G2 cell cycle checkpoint by simaomicin $\alpha$. BBRC 317: 817-822 (2004)

8. Jacobberger JW, Horan PK, Hare JD. Cell cycle analysis of asexual stages of erythrocytic malaria parasites. Cell Prolif 25: 431-445 (1992)

9. Arnot DE, Gul K. The plasmodium cell-cycle: facts and questions. Ann Trop Med Parasitol 92: 361-365 (1998)

10. Lambros C, Vanderberg JP. Synchronization of Plasmodium falciparum erythrocytic stages in culture. J Parasitol 65: 418-420 (1979)

11. Naughton JA, Bell A. Studies on cell-cycle synchronization in the asexual erythrocytic stages of Plasmodium falciparum. Parasitol 134: 331-337 (2007) 